\title{
Analysis of the Viscoelastic Sphere Impact against a Viscoelastic Uflyand-Mindlin Plate considering the Extension of Its Middle Surface
}

\author{
Yury A. Rossikhin, Marina V. Shitikova, and Phan Thanh Trung \\ Research Center for Wave Dynamics of Solids and Structures, Voronezh State Technical University, \\ 20 Letija Oktjabrja Str. 84, Voronezh 394006, Russia \\ Correspondence should be addressed to Marina V. Shitikova; shitikova@vmail.ru
}

Received 7 August 2016; Accepted 13 November 2016; Published 17 January 2017

Academic Editor: Ivo Caliò

Copyright (C) 2017 Yury A. Rossikhin et al. This is an open access article distributed under the Creative Commons Attribution License, which permits unrestricted use, distribution, and reproduction in any medium, provided the original work is properly cited.

In the present paper, the problem on impact of a viscoelastic sphere against a viscoelastic plate is considered with due account for the extension of plate's middle surface and local bearing of sphere and plate's materials via the Hertz theory. The standard linear solid models with conventional derivatives and with fractional-order derivatives are used as viscoelastic models, respectively, outside and within the contact domain. As a result of impact, transient waves (surfaces of strong discontinuity) are generated in the plate, behind the wave fronts of which up to the boundaries of the contact domain the solution is constructed in terms of one-term ray expansions due to short-time duration of the impact process. The motion of the contact zone occurs under the action of extension forces acting in the plate's middle surface, transverse force, and the Hertzian contact force. The suggested approach allows one to find the time-dependence of the impactor's indentation into the target and the Hertzian contact force.

\section{Introduction}

Nowadays fractional calculus is widely used in different fields of science and technology [1-11], including various dynamic problems of mechanics of solids and structures [12]. The problems of impact response of thin-walled structures belong to the most challenging ones among the dynamic problems. The review of papers dealing with the application of fractional calculus models for solving the impact problems could be found in $[12,13]$.

However, to the authors' knowledge, the impact response of viscoelastic plates utilizing the fractional derivative models has been treated only in a few articles. Thus, the problems of impact of an elastic rod or sphere against a viscoelastic Kirchhoff-Love plate or against a viscoelastic UflyandMindlin plate have been considered in [14] and [15-17], respectively. In the case of the classical plate, the method of the Green function construction was used as the method of solution [14]. In the case of the Uflyand-Mindlin plate, the ray method was utilized, according to which the solution behind the fronts of transient waves (surfaces of strong discontinuity) is constructed in terms of truncated power series with variable coefficients. Moreover, the contact force has been taken into account either according to the Hertz contact theory [15] or in terms of linear approximations allowing one to apply Laplace transform technique $[16,17]$.

In all enumerated above papers, the extension of the plate's middle surface, as well as viscoelastic properties of an impactor, has not been considered. However, as experimental research of the behaviour of flexural thin-walled structures under low and middle velocity impacts shows [18], the contribution of extensional deformations into the impact process is rather essential and, therefore, it should be taken into account. Thus, the impact of elastic bodies upon elastic beams and plates with consideration for the transverse deformations and extension of a middle surface was studied in [19], and recently this approach has been considered when investigating the dynamic response of a viscoelastic Timoshenko-type beam impacted by an elastic sphere [20]. 
In the present paper, we will generalize the approaches developed recently in $[20,21]$ when investigating the dynamic response of a viscoelastic beam impacted by an elastic sphere with due account for beam's middle surface extension and by a viscoelastic sphere ignoring the middle surface extension, respectively, over the problem of theoretical analysis of the dynamic response of the viscoelastic plate by considering the influence of the middle surface extension on the process of deformation of the viscoelastic plate during the impact by a viscoelastic spherical impactor.

In both papers $[20,21]$, the viscoelastic properties of the beam have been described by the standard linear solid model with integer time derivatives. During the impact process there occurs decrosslinking within the domain of the contact of the beam with the impactor, resulting in more freely displacements of molecules with respect to each other, and finely in the decrease of the beam material viscosity in the contact zone. This circumstance allows one to describe the behaviour of the beam material within the contact domain by the standard linear solid model involving fractional derivatives, since variation in the fractional parameter (the order of the fractional derivative) enables one to control the viscosity of the beam material.

It is known that viscoelastic properties of the material could be changed during its life-cycle due to different external effects, among them aging and impact loads [22], and/or temperature and irradiation [23]. Dynamic mechanical studies of irradiated polyethylene were presented by Merrill et al. [23], wherein it was found the influence of irradiation on viscoelastic characteristics of polyethylene. Experimental study of concrete aging effect on the contact force and contact time during impact interaction of an elastic rod with a viscoelastic beam was carried out by Popov et al. [22], and a good correlation with the analytical model proposed in [24] was declared.

In the present paper, following $[20,21,24]$ we will study the dynamic response of a viscoelastic Uflyand-Mindlin plate impacted by a viscoelastic sphere considering the extension of plate's middle surface. The standard linear solid model with conventional derivatives and with fractionalorder derivatives will be used as viscoelastic models, respectively, outside and within the contact domain. As a result of impact, transient waves (surfaces of strong discontinuity) are generated in the plate, behind the wave fronts of which up to the boundaries of the contact domain the solution is constructed in terms of one-term ray expansions due to short-time duration of the impact process. The motion of the contact zone is analyzed under the action of extension forces acting in the plate's middle surface, transverse forces, and the Hertzian contact force using the algebra of Rabotnov's fractional operators.

\section{Problem Formulation and Governing Equations}

Let a viscoelastic sphere of radius $R$ and mass $m$ move with the constant velocity $V_{0}$ towards a viscoelastic plate along the $z$-axis, which is perpendicular to the plane of the plate. Impact occurs at $t=0$. As a result of transverse impact against the viscoelastic plate, transient waves, the longitudinal wave, and the wave of transverse shear propagate along the plate, the fronts of which are the surfaces of strong discontinuity. These waves are cylindrical surfaces-strips, the generators of which are parallel to the normal to the median plane, while the guides located in the middle plane are the circumferences extending with the normal velocities $G^{(\alpha)}$. The index $\alpha$ indicates the ordinal number of the wave: $\alpha=1$ for the longitudinal wave and $\alpha=2$ for the transverse wave.

A certain desired function $Z$ behind the fronts of wave surfaces $\Sigma^{(1)}$ and $\Sigma^{(2)}$ could be represented in terms of the ray series [25]

$$
\begin{aligned}
Z(r, t)= & \left.\sum_{\alpha=1}^{2} \sum_{k=0}^{\infty} \frac{1}{k !}\left[Z_{{ }_{(k)}}\right]^{(\alpha)}\right|_{t=r / G^{(\alpha)}}\left(t-\frac{r}{G^{(\alpha)}}\right)^{k} \\
& \cdot H\left(t-\frac{r}{G^{(\alpha)}}\right),
\end{aligned}
$$

where $\left[Z_{{ }_{(k)}}\right]^{(\alpha)}=\left[\partial^{k} Z / \partial t^{k}\right]^{(\alpha)}=Z_{{ }_{(k)}}^{+(\alpha)}-Z_{{ }_{(k)}}^{-(\alpha)}$ are discontinuities in the $k$ th-order time-derivatives of the desired function $Z$ on the waves surfaces $\Sigma_{\alpha}$; that is, at $t=r / G^{(\alpha)}, r$ is the polar radius, the upper indices "+" and "-" denote that the values are calculated immediately ahead of and behind the wave fronts, respectively, and $H\left(t-r / G^{(\alpha)}\right)$ is the unit Heaviside function.

Since the impact process is of short duration, then it is possible to restrict only by zero-order terms, that is,

$$
Z(r, t)=\left.\sum_{\alpha=1}^{2}[Z]^{(\alpha)}\right|_{t=r / G^{(\alpha)}} H\left(t-\frac{r}{G^{(\alpha)}}\right) .
$$

In order to find the discontinuities in the desired values, first it is necessary to write the governing equations describing the dynamic behaviour of the viscoelastic isotropic Uflyand-Mindlin plate in the polar coordinates $(r, \varphi)$

$$
\begin{aligned}
\frac{1}{r}\left(N_{r}-N_{\varphi}\right)+\frac{\partial N_{r}}{\partial r} & =\rho h \ddot{u}_{r}, \\
\frac{1}{r}\left(M_{r}-M_{\varphi}\right)+\frac{\partial M_{r}}{\partial r}+Q_{r} & =\frac{\rho h^{3}}{12} \ddot{\beta}_{r}, \\
\frac{\partial Q_{r}}{\partial r}+\frac{1}{r} Q_{r} & =\rho h \ddot{w}, \\
N_{r} & =\frac{\widetilde{E} h}{1-\widetilde{\sigma}^{2}}\left(\frac{\partial u_{r}}{\partial r}+\widetilde{\sigma} \frac{u_{r}}{r}\right), \\
N_{\varphi} & =\frac{\widetilde{E} h}{1-\widetilde{\sigma}^{2}}\left(\frac{u_{r}}{r}+\widetilde{\sigma} \frac{\partial u_{r}}{\partial r}\right), \\
M_{r} & =\widetilde{D}\left(\frac{\partial \beta_{r}}{\partial r}+\widetilde{\sigma} \frac{\beta_{r}}{r}\right), \\
M_{\varphi} & =\widetilde{D}\left(\frac{\beta_{r}}{r}+\widetilde{\sigma} \frac{\partial \beta_{r}}{\partial r}\right), \\
Q_{r} & =K h \widetilde{\mu}\left(\frac{\partial w_{r}}{\partial r}-\beta_{r}\right),
\end{aligned}
$$


where $N_{r}$ and $N_{\varphi}$ are the forces acting in the plate plane in the $r$ - and $\varphi$-directions, respectively, $M_{r}$ and $M_{\varphi}$ are the bending moments, $Q_{r}$ is the transverse force, $w$ is the plate deflection, $u_{r}$ is the displacement along the radius, $\beta_{r}$ is the rotational angle of the normal in the $r$-direction, $\rho$ is the density, $h$ is the plate thickness, $\widetilde{\mu}$ and $\widetilde{E}$ are shear and Young's operators, respectively, $\widetilde{\sigma}$ is the Poisson's operator, $\widetilde{D}=\widetilde{E} h^{3} / 12\left(1-\widetilde{\sigma}^{2}\right)$ is the operator of the cylindrical rigidity, $K=\pi^{2} / 12$ is the shear coefficient, and overdots denote the time derivatives, and then to apply the kinematic condition of compatibility proposed in [26] for physical components of thin bodies

$$
G\left[\frac{\partial Z}{\partial r}\right]=-[\dot{Z}]+\frac{\delta[Z]}{\delta t}
$$

to (3) and (5), where $\delta / \delta t$ is the Thomas $\delta$-derivative [27] of the function preassigned on the moving surface.

Note that condition (11) is valid in the case when spatial coordinates coincide with the ray coordinates [28].

In order to simplify the procedure for utilizing condition (11), let us interpret the surface of strong discontinuity as a layer of small thickness $\delta$, within which the desired value $Z$ changes monotonically and continuously from the magnitude $Z^{+}$to the magnitude $Z^{-}$. Suppose that the ahead and back fronts of the shock layer arrive at a certain point with the fixed radius at the time instants $t$ and $t+\Delta t$, respectively, where $\Delta t$ is small. Inside the shock layer the following relationship

$$
\frac{\partial Z}{\partial r} \approx-G^{-1} \frac{\partial Z}{\partial t}
$$

is fulfilled.

Writing (3) and (5) within the shock layer and considering (12), we obtain

$$
\begin{aligned}
-G^{-1} \frac{\partial N_{r}}{\partial t} & =\rho h \frac{\partial v_{r}}{\partial t} \\
-G^{-1} \frac{\partial Q_{r}}{\partial t} & =\rho h \frac{\partial W}{\partial t},
\end{aligned}
$$

where $v_{r}=\dot{u}_{r}$, and $W=\dot{w}$.

Thus we have chosen only those equations of motions which will be utilized in further treatment.

Integrating (13) and (14) over $t$ from $t$ to $t+\Delta t$ and then tending $\Delta t \rightarrow 0$ yield

$$
\begin{aligned}
& {\left[N_{r}\right]=-\rho h G\left[v_{r}\right],} \\
& {\left[Q_{r}\right]=-\rho h G[W] .}
\end{aligned}
$$

Relationships (15) and (16) are called the dynamic conditions of compatibility. During the deduction of these conditions we neglect the terms $N_{r}$ and $N_{\varphi}$ in (3) and $Q_{r}$ in (5), as well as the term $\delta Z / \delta t$ in (12), since the integrals from these terms over $t$ from $t$ to $t+\Delta t$ tend to zero as $\Delta t \rightarrow 0$.

In order to write (6) and (10) in discontinuities, it is necessary to decode the operators entering in these equations.

\section{Decoding of Operators}

For decoding the operators involved in (6) and (10), it is a need to specify two any operators, for example, the operator of volume extension-compression $\widetilde{K}$ and shear operator $\widetilde{\mu}$. As numerous experiments with volume stresses and strains show [29], for the majority of materials operator $\widetilde{K}$ is a constant value, that is,

$$
\widetilde{K}=K_{\infty}
$$

where $K_{\infty}$ is a certain constant.

In further treatment the standard linear solid model with conventional derivatives and with fractional-order derivatives will be used as viscoelastic models, respectively, outside and within the contact domain, because during the impact process there occurs decrosslinking within the domain of the contact of the plate with the impactor, resulting in more freely displacements of molecules with respect to each other and finely in the decrease of the plate material viscosity in the contact zone. This circumstance allows us to describe the behaviour of the plate material within the contact domain by the standard linear solid model involving fractional derivatives, since variation in the fractional parameter (the order of the fractional derivative) enables one to control the viscosity of the plate material.

Thus for convenience of presentation, first we will decode fractional-order operators valid inside the contact domain, and then tending fractional parameter to unit in the final relationships we will obtain the corresponding formulae for the viscoelastic plate outside the contact domain in terms of the conventional viscoelasticity.

So within the contact domain of the impactor with the viscoelastic target, we would preassign this connectedness between the deviators of stress $\sigma^{\prime}$ and strain $\varepsilon^{\prime}$ by the standard linear solid model involving fractional derivatives [12], that is,

$$
\sigma^{\prime}+\tau_{\varepsilon}^{\gamma} D^{\gamma} \sigma^{\prime}=2 \mu_{0}\left(\varepsilon^{\prime}+\tau_{\sigma}^{\gamma} D^{\gamma} \varepsilon^{\prime}\right)
$$

or

$$
\sigma^{\prime}=2 \mu_{0} \frac{1+\tau_{\sigma}^{\gamma} D^{\gamma}}{1+\tau_{\varepsilon}^{\gamma} D^{\gamma}} \varepsilon^{\prime},
$$

where $\tau_{\varepsilon}$ and $\tau_{\sigma}$ are the relaxation and retardation times, respectively, $\gamma(0<\gamma \leq 1)$ is the fractional parameter, $\mu_{0}$ is the relaxed shear modulus,

$$
D^{\gamma} x(t)=\frac{d}{d t} \int_{0}^{t} \frac{\left(t-t^{\prime}\right)^{-\gamma}}{\Gamma(1-\gamma)} x\left(t^{\prime}\right) d t^{\prime}
$$

is the Riemann-Liouville fractional derivative of the $\gamma$-order [12], $\Gamma(1-\gamma)$ is the Gamma-function, and $x(t)$ is a certain function.

From (19) it follows that operator $\tilde{\mu}$ is given in the form

$$
\tilde{\mu}=\mu_{0} \frac{1+\tau_{\sigma}^{\gamma} D^{\gamma}}{1+\tau_{\varepsilon}^{\gamma} D^{\gamma}},
$$

or

$$
\tilde{\mu}=\mu_{0} \ni_{\gamma}^{*}\left(\tau_{\varepsilon}^{\gamma}\right)+\mu_{0} \frac{\tau_{\sigma}^{\gamma}}{\tau_{\varepsilon}^{\gamma}} \tau_{\varepsilon}^{\gamma} D^{\gamma} \ni_{\gamma}^{*}\left(\tau_{\varepsilon}\right)
$$


Here the operator

$$
\ni_{\gamma}^{*}\left(\tau_{\varepsilon}^{\gamma}\right)=\frac{1}{1+\tau_{\varepsilon}^{\gamma} D^{\gamma}}
$$

has been introduced, which has the name of the dimensionless Rabotnov fractional operator [13].

If we consider formula

$$
\tau_{\varepsilon}^{\gamma} D^{\gamma} \ni_{\gamma}^{*}\left(\tau_{\varepsilon}^{\gamma}\right)=1-\ni_{\gamma}^{*}\left(\tau_{\varepsilon}^{\gamma}\right)
$$

which is proved by the direct check-up [13], in relationship (22) and introduce the following notation

$$
\mu_{\infty}=\mu_{0} \frac{\tau_{\sigma}^{\gamma}}{\tau_{\varepsilon}^{\gamma}}
$$

where $\mu_{\infty}$ is the nonrelaxed shear modulus, then as a result we obtain

$$
\widetilde{\mu}=\mu_{\infty}\left[1-\nu_{\varepsilon \mu} \ni_{\gamma}^{*}\left(\tau_{\varepsilon}^{\gamma}\right)\right],
$$

where $v_{\varepsilon \mu}=\left(\mu_{\infty}-\mu_{0}\right) \mu_{\infty}^{-1}$ is the dimensionless defect of modulus.

Now let us calculate the Young operator according to the formula

$$
\widetilde{E}=\frac{9 K_{\infty} \widetilde{\mu}}{3 K_{\infty}+\widetilde{\mu}} .
$$

We will start with the operator

$$
3 K_{\infty}+\widetilde{\mu}=\left(3 K_{\infty}+\mu_{\infty}\right)\left[1-M_{\varepsilon} \ni_{\gamma}^{*}\left(\tau_{\varepsilon}^{\gamma}\right)\right],
$$

where $M_{\varepsilon}=\mu_{\infty} v_{\varepsilon \mu}\left(3 K_{\infty}+\mu_{\infty}\right)^{-1}$.

We will seek the inverse operator $\left(3 K_{\infty}+\widetilde{\mu}\right)^{-1}$ in the form

$$
\left(3 K_{\infty}+\tilde{\mu}\right)^{-1}=\left(3 K_{\infty}+\mu_{\infty}\right)^{-1}\left[1+M_{\sigma} \ni_{\gamma}^{*}\left(\tau_{\sigma}^{\gamma}\right)\right],
$$

where $M_{\sigma}$ and $\tau_{\sigma}$ are yet unknown constants.

Further we multiply operators (28) and (29), consider their property $\left(3 K_{\infty}+\widetilde{\mu}\right)\left(3 K_{\infty}+\widetilde{\mu}\right)^{-1}=1$, and utilize the formula

$$
\ni_{\gamma}^{*}\left(\tau_{\varepsilon}^{\gamma}\right) \ni_{\gamma}^{*}\left(\tau_{\sigma}^{\gamma}\right)=\frac{\tau_{\varepsilon}^{\gamma} \ni_{\gamma}^{*}\left(\tau_{\varepsilon}^{\gamma}\right)-\tau_{\sigma}^{\gamma} \ni_{\gamma}^{*}\left(\tau_{\sigma}^{\gamma}\right)}{\tau_{\varepsilon}^{\gamma}-\tau_{\sigma}^{\gamma}},
$$

which is proved by the direct check-up [13].

As a result we obtain

$$
\begin{aligned}
& M_{\sigma} \ni_{\gamma}^{*}\left(\tau_{\sigma}^{\gamma}\right)\left(1+M_{\varepsilon} \frac{\tau_{\sigma}^{\gamma}}{\tau_{\varepsilon}^{\gamma}-\tau_{\sigma}^{\gamma}}\right) \\
& \quad-M_{\varepsilon} \ni_{\gamma}^{*}\left(\tau_{\varepsilon}^{\gamma}\right)\left(1+M_{\sigma} \frac{\tau_{\varepsilon}^{\gamma}}{\tau_{\varepsilon}^{\gamma}-\tau_{\sigma}^{\gamma}}\right)=0 .
\end{aligned}
$$

Equating to zero each bracket in (31), we are led to a set of two equations for defining $M_{\sigma}$ and $\tau_{\sigma}$

$$
\begin{aligned}
& 1+M_{\varepsilon} \frac{\tau_{\sigma}^{\gamma}}{\tau_{\varepsilon}^{\gamma}-\tau_{\sigma}^{\gamma}}=0, \\
& 1+M_{\sigma} \frac{\tau_{\varepsilon}^{\gamma}}{\tau_{\varepsilon}^{\gamma}-\tau_{\sigma}^{\gamma}}=0,
\end{aligned}
$$

whence it follows

$$
\begin{aligned}
M_{\sigma} & =\frac{M_{\varepsilon}}{1-M_{\varepsilon}}, \\
\tau_{\sigma}^{\gamma} & =\frac{\tau_{\varepsilon}^{\gamma}}{1-M_{\varepsilon}}, \\
\frac{\tau_{\varepsilon}^{\gamma}}{\tau_{\sigma}^{\gamma}} & =\frac{M_{\varepsilon}}{M_{\sigma}} .
\end{aligned}
$$

Finally we substitute operators (26) and (29) in (27) and consider relationship (30). As a result we have

$$
\begin{aligned}
\widetilde{E} & =E_{\infty}\left[1-\nu_{\varepsilon \mu} \ni_{\gamma}^{*}\left(\tau_{\varepsilon}^{\gamma}\right)\left(1+M_{\sigma} \frac{\tau_{\varepsilon}^{\gamma}}{\tau_{\varepsilon}^{\gamma}-\tau_{\sigma}^{\gamma}}\right)\right. \\
& \left.+M_{\sigma} \ni_{\gamma}^{*}\left(\tau_{\sigma}^{\gamma}\right)\left(1+\nu_{\varepsilon \mu} \frac{\tau_{\sigma}^{\gamma}}{\tau_{\varepsilon}^{\gamma}-\tau_{\sigma}^{\gamma}}\right)\right] .
\end{aligned}
$$

Considering that according to the set of (32) the first bracket in (34) is equal to zero, while the second bracket is as follows:

$$
1+\nu_{\varepsilon \mu} \frac{\tau_{\sigma}^{\gamma}}{\tau_{\varepsilon}^{\gamma}-\tau_{\sigma}^{\gamma}}=1-\frac{\nu_{\varepsilon \mu}}{M_{\varepsilon}}=-\frac{3 K_{\infty}}{\mu_{\infty}},
$$

and we finally obtain

$$
\widetilde{E}=E_{\infty}\left[1-v_{\varepsilon E_{\gamma}}{ }_{\gamma}^{*}\left(\tau_{\varepsilon E}^{\gamma}\right)\right],
$$

where $v_{\varepsilon E}=M_{\sigma}\left(3 K_{\infty} / \mu_{\infty}\right)=\left(1-\mu_{0} \mu_{\infty}^{-1}\right) /\left(1+\mu_{0}\left(3 K_{\infty}\right)^{-1}\right)<$ 1 , and $\tau_{\varepsilon E}=\tau_{\sigma}$.

Now let us calculate the compliance operator $\widetilde{J}=\widetilde{E}^{-1}$ which is defined in the form

$$
\widetilde{J}=\widetilde{E}^{-1}=E_{\infty}^{-1}\left[1+\nu_{\sigma E} \ni_{\gamma}^{*}\left(\tau_{\sigma E}^{\gamma}\right)\right],
$$

where $\nu_{\sigma E}$ and $\tau_{\sigma E}$ are yet unknown constants. To determine them, we will utilize the property $\widetilde{J} \widetilde{E}=1$ and consider relationship (30). Then we obtain

$$
\begin{aligned}
& -\nu_{\varepsilon E} \ni_{\gamma}^{*}\left(\tau_{\varepsilon E}^{\gamma}\right)\left(1+\nu_{\sigma E} \frac{\tau_{\varepsilon E}^{\gamma}}{\tau_{\varepsilon E}^{\gamma}-\tau_{\sigma E}^{\gamma}}\right) \\
& +\nu_{\sigma E} \ni_{\gamma}^{*}\left(\tau_{\sigma E}^{\gamma}\right)\left(1+\nu_{\varepsilon E} \frac{\tau_{\sigma E}^{\gamma}}{\tau_{\varepsilon E}^{\gamma}-\tau_{\sigma E}^{\gamma}}\right)=0 .
\end{aligned}
$$

Equating to zero each bracket in (38) yields

$$
\begin{aligned}
& 1+\nu_{\sigma E} \frac{\tau_{\varepsilon E}^{\gamma}}{\tau_{\varepsilon E}^{\gamma}-\tau_{\sigma E}^{\gamma}}=0, \\
& 1+\nu_{\varepsilon E} \frac{\tau_{\sigma E}^{\gamma}}{\tau_{\varepsilon E}^{\gamma}-\tau_{\sigma E}^{\gamma}}=0 .
\end{aligned}
$$

Solving the set of (39), we find

$$
\begin{aligned}
& \left(\frac{\tau_{\sigma E}}{\tau_{\varepsilon E}}\right)^{\gamma}=1+v_{\sigma E} \\
& \left(\frac{\tau_{\varepsilon E}}{\tau_{\sigma E}}\right)^{\gamma}=1-v_{\varepsilon E} .
\end{aligned}
$$


Now we could calculate the Poisson's operator $\widetilde{\sigma}$ according to formula (17), which could be rewritten in the form

$$
\frac{\widetilde{E}}{1-2 \widetilde{\sigma}}=\frac{E_{\infty}}{1-2 \sigma_{\infty}}=3 K_{\infty} .
$$

Considering (36), from formula (41) we have

$$
\widetilde{\sigma}=\sigma_{\infty}+\frac{1}{2}\left(1-2 \sigma_{\infty}\right) \nu_{\varepsilon E_{\gamma}}^{*}\left(\tau_{\varepsilon E}^{\gamma}\right)
$$

For further treatment we should know the following operators:

$$
\begin{aligned}
& \frac{1}{1+\widetilde{\sigma}}=\frac{1}{1+\sigma_{\infty}+(1 / 2)\left(1-2 \sigma_{\infty}\right) \nu_{\varepsilon E} \ni_{\gamma}^{*}\left(\tau_{\varepsilon E}^{\gamma}\right)}, \\
& \frac{1}{1-\widetilde{\sigma}}=\frac{1}{1-\sigma_{\infty}-(1 / 2)\left(1-2 \sigma_{\infty}\right) \nu_{\varepsilon E_{\gamma} \vartheta^{*}}\left(\tau_{\varepsilon E}^{\gamma}\right)} \text {. }
\end{aligned}
$$

In order to calculate the operators in the right-hand side of (43) and (44), we assume that they have the following form:

$$
\begin{aligned}
& \frac{1}{1+\tilde{\sigma}}=\frac{1}{1+\sigma_{\infty}}\left[1-B \ni_{\gamma}^{*}\left(t_{1}^{\gamma}\right)\right], \\
& \frac{1}{1-\tilde{\sigma}}=\frac{1}{1-\sigma_{\infty}}\left[1+D \ni_{\gamma}^{*}\left(t_{2}^{\gamma}\right)\right],
\end{aligned}
$$

where $B, t_{1}$ and $D, t_{2}$ are yet unknown constants.

Equating the right sides of relationships (43), (45) and (44), (46), reducing the obtained expressions to the common denominator, and considering (30) yield

$$
\begin{aligned}
& \frac{1}{2} \frac{\left(1-2 \sigma_{\infty}\right) v_{\varepsilon E}}{1+\sigma_{\infty}}\left[1-B \frac{\tau_{\varepsilon E}^{\gamma}}{\tau_{\varepsilon E}^{\gamma}-t_{1}^{\gamma}}\right] \ni_{\gamma}^{*}\left(\tau_{\varepsilon E}^{\gamma}\right) \\
& -B\left[1-\frac{1}{2} \frac{\left(1-2 \sigma_{\infty}\right) v_{\varepsilon E}}{\left(1+\sigma_{\infty}\right)} \frac{t_{1}^{\gamma}}{\left(\tau_{\varepsilon E}^{\gamma}-t_{1}^{\gamma}\right)}\right] \ni_{\gamma}^{*}\left(t_{1}^{\gamma}\right)=0, \\
& -\frac{1}{2} \frac{\left(1-2 \sigma_{\infty}\right) v_{\varepsilon E}}{1-\sigma_{\infty}}\left[1+D \frac{\tau_{\varepsilon E}^{\gamma}}{\tau_{\varepsilon E}^{\gamma}-t_{2}^{\gamma}}\right] \ni_{\gamma}^{*}\left(\tau_{\varepsilon E}^{\gamma}\right) \\
& +D\left[1+\frac{1}{2} \frac{\left(1-2 \sigma_{\infty}\right) v_{\varepsilon E}}{\left(1-\sigma_{\infty}\right)} \frac{t_{2}^{\gamma}}{\left(\tau_{\varepsilon E}^{\gamma}-t_{2}^{\gamma}\right)}\right] \ni_{\gamma}^{*}\left(t_{2}^{\gamma}\right)=0 .
\end{aligned}
$$

Vanishing to zero the expressions in square brackets in (47), we determine unknown constants

$$
\begin{aligned}
B & =\frac{\left(1-2 \sigma_{\infty}\right) \nu_{\varepsilon E}}{2\left(1+\sigma_{\infty}\right)+\left(1-2 \sigma_{\infty}\right) v_{\varepsilon E}}=\frac{\tau_{\varepsilon E}^{\gamma}-t_{1}^{\gamma}}{\tau_{\varepsilon E}^{\gamma}}, \\
t_{1}^{-\gamma} & =\tau_{\varepsilon E}^{-\gamma}\left[1+\frac{\left(1-2 \sigma_{\infty}\right) \nu_{\varepsilon E}}{2\left(1+\sigma_{\infty}\right)}\right], \\
t_{1}^{\gamma} & =\frac{\tau_{\varepsilon E}^{\gamma}}{A}, \\
D & =\frac{\left(1-2 \sigma_{\infty}\right) v_{\varepsilon E}}{2\left(1-\sigma_{\infty}\right)-\left(1-2 \sigma_{\infty}\right) v_{\varepsilon E}}=\frac{\tau_{\varepsilon E}^{\gamma}-t_{2}^{\gamma}}{\tau_{\varepsilon E}^{\gamma}},
\end{aligned}
$$

$$
\begin{aligned}
t_{2}^{-\gamma} & =\tau_{\varepsilon E}^{-\gamma}\left[1-\frac{\left(1-2 \sigma_{\infty}\right) v_{\varepsilon E}}{2\left(1-\sigma_{\infty}\right)}\right], \\
t_{2}^{\gamma} & =\frac{\tau_{\varepsilon E}^{\gamma}}{C}, \\
A & =\frac{2\left(1+\sigma_{\infty}\right)+\left(1-2 \sigma_{\infty}\right) v_{\varepsilon E}}{2\left(1+\sigma_{\infty}\right)}, \\
C & =\frac{2\left(1-\sigma_{\infty}\right)-\left(1-2 \sigma_{\infty}\right) v_{\varepsilon E}}{2\left(1-\sigma_{\infty}\right)} .
\end{aligned}
$$

Now we could calculate the operator

$$
\frac{\widetilde{E}}{1-\widetilde{\sigma}^{2}}=\frac{\widetilde{E}}{2}\left(\frac{1}{1+\widetilde{\sigma}}+\frac{1}{1-\widetilde{\sigma}}\right) .
$$

For this purpose, we substitute (36), (45), and (46) in (49) with due account for (30). As a result we obtain

$$
\frac{\widetilde{E}}{1-\widetilde{\sigma}^{2}}=\frac{E_{\infty}}{1-\sigma_{\infty}^{2}}\left[1-m_{1} \ni_{\gamma}^{*}\left(t_{1}^{\gamma}\right)-m_{2} \ni_{\gamma}^{*}\left(t_{2}^{\gamma}\right)\right],
$$

where

$$
\begin{aligned}
& m_{1}=\frac{3}{2} \frac{B\left(1-\sigma_{\infty}\right)}{\left(1-2 \sigma_{\infty}\right)}, \\
& m_{2}=\frac{1}{2} \frac{D\left(1+\sigma_{\infty}\right)}{\left(1-2 \sigma_{\infty}\right)} .
\end{aligned}
$$

Finally we would calculate operator $\widetilde{E} \widetilde{\sigma}\left(1-\widetilde{\sigma}^{2}\right)^{-1}$ considering formulas (30) and (48)

$$
\frac{\widetilde{E}}{1-\widetilde{\sigma}^{2}} \widetilde{\sigma}=\frac{E_{\infty} \sigma_{\infty}}{1-\sigma_{\infty}^{2}}\left[1+\frac{m_{1}}{\sigma_{\infty}} \ni_{\gamma}^{*}\left(t_{1}^{\gamma}\right)-\frac{m_{2}}{\sigma_{\infty}} \ni_{\gamma}^{*}\left(t_{2}^{\gamma}\right)\right] .
$$

Further for the convenience we will represent the Rabotnov fractional operator in another form multiplying the numerator and denominator of (23) by $\tau_{\varepsilon}^{-\gamma} I^{\gamma}$, where

$$
I^{\gamma} x(t)=\int_{0}^{t} \frac{\left(t-t^{\prime}\right)^{\gamma-1}}{\Gamma(\gamma)} x\left(t^{\prime}\right) d t^{\prime}
$$

is the fractional integral.

As a result we obtain

$$
\ni_{\gamma}^{*}\left(\tau_{i}^{\gamma}\right)=\frac{I^{\gamma} \tau_{i}^{-\gamma}}{1-\left(-I^{\gamma} \tau_{i}^{-\gamma}\right)}, \quad(i=\varepsilon, \sigma)
$$

It has been shown in [13] that interpreting the righthand side part of (54) as a sum of the infinite descending geometrical progression with the denominator $d=-I^{\gamma} \tau_{i}^{-\gamma}$, we have

$$
\ni_{\gamma}^{*}\left(\tau_{i}^{\gamma}\right)=\sum_{n=0}^{\infty}(-1)^{n} \tau_{i}^{-\gamma(n+1)} I^{\gamma(n+1)}
$$


or

$$
\ni_{\gamma}^{*}\left(\tau_{i}^{\gamma}\right) x(t)=\int_{0}^{t} \ni_{\gamma}\left(-\frac{t-t^{\prime}}{\tau_{i}}\right) x\left(t^{\prime}\right) d t^{\prime},
$$

where

$$
\ni_{\gamma}\left(-\frac{t}{\tau_{i}}\right)=\frac{t^{\gamma-1}}{\tau_{i}^{\gamma}} \sum_{n=0}^{\infty}(-1)^{n} \frac{\left(t / \tau_{i}\right)^{\gamma n}}{\Gamma[\gamma(n+1)]}
$$

is Rabotnov's fractional exponential function [29, 30], which at $\gamma=1$ goes over into the conventional exponent, that is,

$$
\ni_{1}^{*}\left(\tau_{i}\right) x(t)=\frac{1}{\tau_{i}} \int_{0}^{t} \exp \left(-\frac{t-t^{\prime}}{\tau_{i}}\right) x\left(t^{\prime}\right) d t^{\prime} .
$$

All formulas which have been obtained for the viscoelastic fractional derivative standard linear solid model remain valid for the standard linear solid model with conventional derivatives.

Since out of the contact domain viscoelastic features of plate's material are described via the standard linear solid model with conventional derivatives, then it is possible to rewrite (6) and (10) with due account for the derived above operators putting $\gamma=1$. As a result we have

$$
\begin{aligned}
N_{r}= & \frac{E_{\infty} h}{1-\sigma_{\infty}^{2}}\left[1-m_{1} \ni_{1}^{*}\left(t_{1}\right)-m_{2} \ni_{1}^{*}\left(t_{2}\right)\right] \frac{\partial u_{r}}{\partial r} \\
& +\frac{E_{\infty} h \sigma_{\infty}}{1-\sigma_{\infty}^{2}}\left[1+\frac{m_{1}}{\sigma_{\infty}} \ni_{1}^{*}\left(t_{1}\right)-\frac{m_{2}}{\sigma_{\infty}} \ni_{1}^{*}\left(t_{2}\right)\right] \frac{u_{r}}{r}, \\
Q_{r}= & K h \mu_{\infty}\left[1-v_{\varepsilon \mu} \ni_{1}^{*}\left(\tau_{\varepsilon}\right)\right]\left(\frac{\partial w}{\partial r}-\beta_{r}\right) .
\end{aligned}
$$

In order to write relationships (59) in discontinuities, let us substitute there $\partial u_{r} / \partial r$ and $\partial w / \partial r$ by $-G^{-1} v_{r}$ and $-G^{-1} W$, respectively, according to (12), and then write the obtained relationships at the moments $t$ and $t+\Delta t$. Thus,

$$
\begin{aligned}
& N_{r}^{+}=-\frac{E_{\infty} h G^{-1}}{1-\sigma_{\infty}^{2}}\left[v_{r}^{+}-\frac{m_{1}}{t_{1}} \int_{0}^{t} e^{-\left(t-t^{\prime}\right) / t_{1}} v_{r}\left(t^{\prime}\right) d t^{\prime}\right. \\
& \left.-\frac{m_{2}}{t_{2}} \int_{0}^{t} e^{-\left(t-t^{\prime}\right) / t_{2}} v_{r}\left(t^{\prime}\right) d t^{\prime}\right]+\frac{E_{\infty} h \sigma_{\infty}}{\left(1-\sigma_{\infty}^{2}\right) r}\left[u_{r}^{+}\right. \\
& +\frac{m_{1}}{\sigma_{\infty} t_{1}} \int_{0}^{t} e^{-\left(t-t^{\prime}\right) / t_{1}} u_{r}\left(t^{\prime}\right) d t^{\prime}-\frac{m_{2}}{\sigma_{\infty} t_{2}} \\
& \left.\cdot \int_{0}^{t} e^{-\left(t-t^{\prime}\right) / t_{2}} u_{r}\left(t^{\prime}\right) d t^{\prime}\right] \\
& N_{r}^{-}=-\frac{E_{\infty} h G^{-1}}{1-\sigma_{\infty}^{2}}\left[v_{r}^{-}-\frac{m_{1}}{t_{1}}\right. \\
& \cdot \int_{0}^{t+\Delta t} e^{-\left(t+\Delta t-t^{\prime}\right) / t_{1}} v_{r}\left(t^{\prime}\right) d t^{\prime}-\frac{m_{2}}{t_{2}}
\end{aligned}
$$

$$
\begin{gathered}
\left.\cdot \int_{0}^{t+\Delta t} e^{-\left(t+\Delta t-t^{\prime}\right) / t_{2}} v_{r}\left(t^{\prime}\right) d t^{\prime}\right]+\frac{E_{\infty} h \sigma_{\infty}}{\left(1-\sigma_{\infty}^{2}\right) r}\left[u_{r}^{-}\right. \\
+\frac{m_{1}}{\sigma_{\infty} t_{1}} \int_{0}^{t+\Delta t} e^{-\left(t+\Delta t-t^{\prime}\right) / t_{1}} u_{r}\left(t^{\prime}\right) d t^{\prime}-\frac{m_{2}}{\sigma_{\infty} t_{2}} \\
\left.\cdot \int_{0}^{t+\Delta t} e^{-\left(t+\Delta t-t^{\prime}\right) / t_{2}} u_{r}\left(t^{\prime}\right) d t^{\prime}\right] \\
Q_{r}^{+}=-K h \mu_{\infty}\left[\left(G^{-1} W^{+}+\beta_{r}^{+}\right)-\frac{v_{\varepsilon \mu}}{\tau_{\varepsilon}}\right. \\
\left.\cdot \int_{0}^{t} e^{-\left(t-t^{\prime}\right) / \tau_{\varepsilon}}\left(G^{-1} W\left(t^{\prime}\right)+\beta_{r}\left(t^{\prime}\right)\right) d t^{\prime}\right], \\
Q_{r}^{-}=-K h \mu_{\infty}\left[\left(G^{-1} W^{-}+\beta_{r}^{-}\right)-\frac{v_{\varepsilon \mu}}{\tau_{\varepsilon}}\right. \\
\left.\cdot \int_{0}^{t+\Delta t} e^{-\left(t+\Delta t-t^{\prime}\right) / \tau_{\varepsilon}}\left(G^{-1} W\left(t^{\prime}\right)+\beta_{r}\left(t^{\prime}\right)\right) d t^{\prime}\right] .
\end{gathered}
$$

Expanding the integrals entering in (61) and (63) in Taylor series in terms of the small parameter $\Delta t$ and limiting ourselves by the zero and first approximations yield

$$
\begin{aligned}
\int_{0}^{t+\Delta t} & e^{-\left(t+\Delta t-t^{\prime}\right) / t_{i}} x\left(t^{\prime}\right) d t^{\prime} \\
= & \int_{0}^{t} e^{-\left(t-t^{\prime}\right) / t_{i}} x\left(t^{\prime}\right) d t^{\prime}+x(t) \Delta t \\
& -\frac{\Delta t}{t_{i}} \int_{0}^{t} e^{-\left(t-t^{\prime}\right) / t_{i}} x\left(t^{\prime}\right) d t^{\prime}, \quad(i=1,2,3),
\end{aligned}
$$

where $x(t)$ may coincide with $v_{r}(t), u_{r}(t)$, or $G^{-1} W(t)+\beta_{r}(t)$, and $t_{3}=\tau_{\varepsilon}$.

Subtracting (61) from (60) and (63) from (62), considering relationship (64) as well as that $u_{r}^{+}=u_{r}^{-}, \beta_{r}^{+}=\beta_{r}^{-}$, $v_{r}^{+}-v_{r}^{-}=\left[v_{r}\right], W^{+}-W^{-}=[W], N_{r}^{+}-N_{r}^{-}=\left[N_{r}\right]$, $Q_{r}^{+}-Q_{r}^{-}=\left[Q_{r}\right]$, and tending $\Delta t$ to zero, we have

$$
\begin{aligned}
& {\left[N_{r}\right]=-\frac{E_{\infty} h}{\left(1-\sigma_{\infty}^{2}\right) G}\left[v_{r}\right],} \\
& {\left[Q_{r}\right]=-\frac{K h \mu_{\infty}}{G}[W] .}
\end{aligned}
$$

From expressions (15) and (65), (16), and (66) it is possible to find velocities of two types of transient waves, namely, longitudinal-flexural wave and transverse shear wave:

$$
\begin{aligned}
& G_{\infty}^{(1)}=\sqrt{\frac{E_{\infty}}{\rho\left(1-\sigma_{\infty}^{2}\right)}}, \\
& G_{\infty}^{(2)}=\sqrt{\frac{K \mu_{\infty}}{\rho}} .
\end{aligned}
$$


Substituting the found velocities (67) and (68) in formulas (15) and (16), and limiting, as it has been already mentioned, by the zeroth terms of the ray expansions, we obtain

$$
\begin{aligned}
& N_{r}=-\rho h G_{\infty}^{(1)} v_{r}, \\
& Q_{r}=-\rho h G_{\infty}^{(2)} W .
\end{aligned}
$$

Relationships (69) and (70) by their form do not differ from the corresponding relationships for an elastic plate, with the only difference that a subindex $\infty$ has appeared at the velocity notation, which indicates that the velocities are expressed in terms of the nonrelaxed modulus of the viscoelastic model (see Appendix).

\section{Equations of Motion of the Contact Domain and the Impactor}

At $t>0$ the displacement of the sphere's center $z(t)$ could be represented in the form

$$
z=w+\alpha,
$$

where $\alpha$ is the impactor's indentation due to the local bearing of target and impactors' materials within the contact domain.

Then the equation of motion of the part of the plate being in contact with the sphere and the equation of the sphere have the form

$$
\begin{aligned}
2 \pi a N_{r} \frac{\partial w}{\partial r}+2 \pi a Q_{r}+P(t) & =\rho h \pi a^{2} \ddot{w}, \\
m \ddot{z} & =-P(t),
\end{aligned}
$$

where $a(t)$ is the radius of the contact domain, and $P(t)$ is the contact force.

Equations (72) and (73) could be solved with due account for formulas (69) and (70), as well as considering the following initial conditions:

$$
\begin{aligned}
& \left.z\right|_{t=0}=0, \\
& \left.\dot{z}\right|_{t=0}=V_{0}, \\
& \left.w\right|_{t=0}=0, \\
& \left.\dot{w}\right|_{t=0}=0 .
\end{aligned}
$$

The relationships

$$
\begin{aligned}
P(t) & =\frac{4 \sqrt{R}}{3} \widetilde{k} \alpha^{3 / 2}, \\
a & =\sqrt{\alpha R}
\end{aligned}
$$

following from the generalized contact Hertz theory should be added to (72) and (73), where

$$
\widetilde{k}=\left(\frac{1-\tilde{\sigma}_{1}^{2}}{\widetilde{E}_{1}}+\frac{1-\tilde{\sigma}_{2}^{2}}{\widetilde{E}_{2}}\right)^{-1},
$$

and in doing so the indices " 1 " and " 2 " refer to the viscoelastic plate and viscoelastic sphere, respectively. The operators valid within the contact domain are based on the fractional derivative standard linear solid models.

Integrating (73) yields

$$
z=-\frac{1}{m} \int_{0}^{t} P\left(t^{\prime}\right)\left(t-t^{\prime}\right) d t^{\prime}+V_{0} t
$$

In order to express the contact force $P(t)$ in terms of displacement $\alpha(t)$, it is needed to decode the operator $\widetilde{k}$. The procedure for decoding this operator as well as the construction of its inverse operator has been recently described in detail in Rossikhin et al. [21]. Thus, it has been shown [21] that operator $\widetilde{k}$ could be written in the form

$$
\tilde{k}=æ_{\infty}^{-1}\left[1-\sum_{j=1}^{4} M_{j} \ni_{\gamma}^{*}\left(T_{j}^{\gamma}\right)\right],
$$

where

$$
æ_{\infty}=\frac{1-\sigma_{1 \infty}^{2}}{E_{1 \infty}}+\frac{1-\sigma_{2 \infty}^{2}}{E_{2 \infty}},
$$

and unknown constants $M_{j}$ and $T_{j}$ could be determined from the set of equations

$$
\begin{aligned}
& 1+\sum_{k=1}^{4} \frac{n_{k} \tau_{k}^{-\gamma}}{\tau_{k}^{-\gamma}-T_{j}^{-\gamma}}=0, \\
& 1+\sum_{j=1}^{4} \frac{M_{j} T_{j}^{-\gamma}}{\tau_{k}^{-\gamma}-T_{j}^{-\gamma}}=0,
\end{aligned}
$$

wherein

$$
\begin{aligned}
& \tau_{1}^{\gamma}=\tau_{1 \varepsilon E}^{\gamma}, \\
& \tau_{2}^{\gamma}=\tau_{1 \sigma E}^{\gamma}, \\
& \tau_{3}^{\gamma}=\tau_{2 \varepsilon E}^{\gamma}, \\
& \tau_{4}^{\gamma}=\tau_{2 \sigma E}^{\gamma}, \\
& n_{1}=\frac{\left(1-2 \sigma_{1 \infty}\right)^{2} \nu_{1 \varepsilon E}}{4 E_{1 \infty} æ_{\infty}}, \\
& n_{2}=\frac{3 v_{1 \sigma E}}{4 E_{1 \infty} æ_{\infty}}, \\
& n_{3}=\frac{\left(1-2 \sigma_{2 \infty}\right)^{2} \nu_{2 \varepsilon E}}{4 E_{2 \infty} æ_{\infty}}, \\
& n_{4}=\frac{3 v_{2 \sigma E}}{4 E_{2 \infty} æ_{\infty}} .
\end{aligned}
$$

Considering (79), the contact force is defined as

$$
\begin{aligned}
P(t) & =k_{\infty}\left[\alpha^{3 / 2}(t)\right. \\
& \left.-\sum_{j=1}^{4} M_{j} \int_{0}^{t} \ni_{\gamma}\left(-\frac{t-t^{\prime \prime}}{T_{j}}\right) \alpha^{3 / 2}\left(t^{\prime \prime}\right) d t^{\prime \prime}\right],
\end{aligned}
$$

where $k_{\infty}=4 \sqrt{R} / 3 æ_{\infty}$. 
Utilizing (83), it is possible to rewrite (78) in the form

$$
\begin{aligned}
z(t) & =V_{0} t-\frac{k_{\infty}}{m} \int_{0}^{t}\left[\alpha^{3 / 2}\left(t^{\prime}\right)\right. \\
& \left.-\sum_{j=1}^{4} M_{j} \int_{0}^{t^{\prime}} \ni_{\gamma}\left(-\frac{t^{\prime}-t^{\prime \prime}}{T_{j}}\right) \alpha^{3 / 2}\left(t^{\prime \prime}\right) d t^{\prime \prime}\right](t \\
& \left.-t^{\prime}\right) d t^{\prime} .
\end{aligned}
$$

The values of $N_{r}$ and $Q_{r}$ defined by (69) and (70), respectively, should be substituted in (72); then this equation together with (84) will give us a set of two governing equations involving only two unknown values, $\alpha$ and $w$. However, the force $N_{r}$ entering in (72) depends on the velocity $v_{r}$ according to (69); thus it is necessary to express it in terms of $\alpha$ and $w$.

With this purpose in mind, we write the stress tensor $\sigma_{i j}$ for a three-dimensional viscoelastic medium

$$
\sigma_{i j}=\tilde{\lambda} u_{l, l} \delta_{i j}+\tilde{\mu}\left(u_{i, j}+u_{j, i}\right),
$$

where $u_{i}$ are components of the displacement vector, summation is carried out over two repeated indices, and $\delta_{i j}$ is the Kronecker symbol, $i, j=1,2,3$, an index after a comma labels the derivative with respect to the coordinates $x_{1}=x, x_{2}=y$, and $x_{3}=z$.

Considering formula (26), as well as the expression for operator

$$
\tilde{\lambda}=\lambda_{\infty}\left[1+\nu_{\varepsilon \lambda} \ni_{\gamma}^{*}\left(\tau_{\varepsilon}^{\gamma}\right)\right]
$$

where $v_{\varepsilon \lambda}=(2 / 3)\left(\mu_{\infty} / \lambda_{\infty}\right) v_{\varepsilon \mu}$, which follows from the formula

$$
K_{\infty}=\tilde{\lambda}+\frac{2}{3} \tilde{\mu},
$$

let us rewrite (85) in the form

$$
\begin{aligned}
\sigma_{i j}= & \lambda_{\infty}\left[1+\nu_{\varepsilon \lambda} \ni_{\gamma}^{*}\left(\tau_{\varepsilon}^{\gamma}\right)\right] u_{l, l} \delta_{i j} \\
& +\mu_{\infty}\left[1-v_{\varepsilon \mu} \ni_{\gamma}^{*}\left(\tau_{\varepsilon}^{\gamma}\right)\right]\left(u_{i, j}+u_{j, i}\right) .
\end{aligned}
$$

Using the procedure applied above for deducing (65) and (66), from (88) we obtain

$$
\left[\sigma_{i j}\right]=\lambda_{\infty}\left[u_{l, l}\right] \delta_{i j}+\mu_{\infty}\left(\left[u_{i, j}\right]+\left[u_{j, i}\right]\right)
$$

Applying the geometric condition of compatibility

$$
G\left[u_{j, i}\right]=-\left[v_{j}\right] v_{i}+G\left[u_{j, N}\right] N_{i},
$$

where $v_{i}$ are the components of the unit vector $\vec{v}$ normal to the wave surface, and $N_{i}$ are the components of the unit vector $\vec{N}$ normal to the plate, to (89), yields

$$
\begin{aligned}
0= & -\lambda_{\infty} G^{-1}\left[v_{l}\right] v_{l} N_{i}-\mu_{\infty} G^{-1}\left[v_{j}\right] N_{j} v_{i}+\mu_{\infty}\left[u_{i, N}\right] \\
& +\left(\lambda_{\infty}+\mu_{\infty}\right)\left[u_{N, N}\right] N_{i},
\end{aligned}
$$

where $\left[u_{i, N}\right]=\left[u_{i, j}\right] N_{j}$, and $\left[u_{N, N}\right]=\left[u_{i, j}\right] N_{i} N_{j}$.
Note that condition (90) differs from the Hadamard condition of compatibility, since (90) involves the second term taking the transverse shear deformation of the plate into account.

Multiplying (91) by $N_{i}$, we find

$$
G^{-1}\left[v_{l}\right] \nu_{l}=\left(\lambda_{\infty}+2 \mu_{\infty}\right) \lambda_{\infty}^{-1}\left[u_{N, N}\right]
$$

Considering that for the cylindrical wave-strip propagating in the plate $\left[v_{l}\right] v_{l}=\left[v_{r}\right]$ and $\left[u_{N, N}\right]=\left[u_{z, z}\right]$ in the cylindrical set of coordinates $(r, \varphi, z)$ and that $\lambda_{\infty}\left(\lambda_{\infty}+\right.$ $\left.\mu_{\infty}\right)^{-1}=\sigma_{\infty}\left(1-\sigma_{\infty}\right)^{-1}$, we are led to the following relationship:

$$
G_{\infty}^{(1)}\left[u_{z, z}\right]=\sigma_{\infty}\left(1-\sigma_{\infty}\right)^{-1}\left[v_{r}\right],
$$

wherein the value $\left[u_{z, z}\right]$, in its turn, is defined by the formula

$$
\left[u_{z, z}\right]=-\frac{\alpha}{h} .
$$

Then the discontinuity $\left[v_{r}\right]$ could be written as

$$
\left[v_{r}\right]=-\frac{G_{\infty}^{(1)} \alpha\left(1-\sigma_{\infty}\right)}{h \sigma_{\infty}},
$$

and the value $N_{r}$ due to (69) takes the form

$$
N_{r}=\frac{\rho G_{\infty}^{(1)^{2}} \alpha\left(1-\sigma_{\infty}\right)}{\sigma_{\infty}} .
$$

Considering formulas (70), (76), (83), and (96), as well as the relationship

$$
\frac{\partial w}{\partial r}=-G_{\infty}^{(2)^{-1}} W
$$

which is obtained from formula (12), if we substitute there the function $Z$ by the function $w$, we could rewrite (72) in the form

$$
\begin{aligned}
& M \alpha \dot{W}=-e \alpha^{3 / 2} W-g \alpha^{1 / 2} W+k_{\infty}\left[\alpha^{3 / 2}\right. \\
& \left.-\sum_{j=1}^{4} M_{j} \int_{0}^{t} \ni_{\gamma}\left(-\frac{t-t^{\prime \prime}}{T_{j}}\right) \alpha^{3 / 2}\left(t^{\prime \prime}\right) d t^{\prime \prime}\right],
\end{aligned}
$$

where

$$
\begin{aligned}
e & =\frac{2 G_{\infty}^{(1)^{2}}\left(1-\sigma_{\infty}\right) M}{h \sqrt{R} G_{\infty}^{(2)} \sigma_{\infty}}, \\
g & =\frac{2 G_{\infty}^{(2)} M}{\sqrt{R}} \\
M & =\rho h \pi R .
\end{aligned}
$$

Note that since the impact process is of short duration, then in the integrals entering in (84) and (98)

$$
\ni_{\gamma}\left(-\frac{t}{T_{j}}\right) \approx \frac{t^{\gamma-1}}{T_{j}^{\gamma} \Gamma(\gamma)}, \quad(j=1,2,3,4) .
$$


Consider (100) and (84) and (98) are reduced to

$$
\begin{aligned}
& z(t)=V_{0} t-\frac{k_{\infty}}{m} \int_{0}^{t}\left[\alpha^{3 / 2}\left(t^{\prime}\right)\right. \\
& \left.-\sum_{j=1}^{4} M_{j} \int_{0}^{t^{\prime}} \frac{\left(t^{\prime}-t^{\prime \prime}\right)^{\gamma-1}}{T_{j}^{\gamma} \Gamma(\gamma)} \alpha^{3 / 2}\left(t^{\prime \prime}\right) d t^{\prime \prime}\right](t \\
& \left.-t^{\prime}\right) d t^{\prime}, \\
& M \alpha \dot{W}=-\left(e \alpha^{3 / 2}+g \alpha^{1 / 2}\right) W+k_{\infty}\left[\alpha^{3 / 2}(t)\right. \\
& \left.-\sum_{j=1}^{4} M_{j} \int_{0}^{t} \frac{\left(t-t^{\prime \prime}\right)^{\gamma-1}}{T_{j}^{\gamma} \Gamma(\gamma)} \alpha^{3 / 2}\left(t^{\prime \prime}\right) d t^{\prime \prime}\right] .
\end{aligned}
$$

Adding formula (71) to (101) and (102), we obtain the closed set of three equations in three unknown functions, namely, $z(t), w(t)$, and $\alpha(t)$.

\section{Approximate Analytical Solution of the Governing Set of Equations}

Now we will consider several particular cases.

5.1. The Case $\gamma=0$. Let us consider first the case when $\gamma=0$ which corresponds to an elastic target and elastic impactor.

Since at $\gamma=0$

$$
\sum_{j=1}^{4} M_{j}=0
$$

as it has been shown in [21], then in (101) and (102) the terms involving the sums vanish to zero; therefore, (102) is reduced to

$$
M \alpha \dot{W}=-\alpha^{1 / 2}(e \alpha+g) W+k_{\infty} \alpha^{3 / 2},
$$

while (101) could be retained in the form of (73).

If we neglect the inertia of the contact domain due to its smallness, then it could be rewritten as

$$
W=k_{\infty} \frac{\alpha}{e \alpha+g} .
$$

Consider relationships

$$
\begin{aligned}
\dot{W} & =\frac{d W}{d \alpha} \dot{\alpha}, \\
\frac{d W}{d \alpha} & =\frac{k_{\infty} g}{(e \alpha+g)^{2}},
\end{aligned}
$$

and introduce a new variable

$$
A=\dot{\alpha}
$$

Equation (73) with due account of (105) could be rewritten in the form

$$
A \frac{d A}{d \alpha}+\frac{k_{\infty} g}{(e \alpha+g)^{2}} A=-\frac{k_{\infty}}{m} \alpha^{3 / 2},
$$

where $A(d A / d \alpha)=\ddot{\alpha}$.

If we ignore the deformation of the middle surface, that is, putting $e=0$, then (108) is reduced to

$$
A \frac{d A}{d \alpha}+a_{1} A=-\frac{k_{\infty}}{m} \alpha^{3 / 2}
$$

where $a_{1}=k_{\mathrm{o}} / g$.

Note that (109) is the Abel equation of the second kind [31], and its solution was found in $[25,32]$ in the form

$$
A=V_{0}-a_{1} \alpha+a_{2} \alpha^{5 / 2}+\sum_{n=2}^{\infty} a_{n+1} \alpha^{(2 n+3) / 2}
$$

where

$$
a_{n+1}=\prod_{m=2}^{n}\left(\frac{2 m+1}{2 m+3}\right)\left(\frac{a_{1}}{V_{0}}\right)^{n-1} a_{2}, \quad a_{2}=-\frac{2}{5} \frac{k_{\infty}}{V_{0} m} .
$$

It has been emphasized in $[25,32]$ that coefficients $a_{1}$ and $a_{2}$ define two different processes being caused by the shock interaction of the impactor and the target: the coefficient $a_{1}$ is responsible for the dynamic processes generating in the plate during propagation of the surfaces of the discontinuity, while the coefficient $a_{2}$ describes the quasistatic processes occurring at the local bearing of the material due to Hertzian theory. If $a_{1} \rightarrow 0$, what corresponds to an infinite large velocity of transverse shear wave propagation (KirchhoffLove plate), then solution (110) for small $\alpha$ goes over into the quasistatic solution [18].

If $e \rightarrow \infty$ in (108), then we are led to the well-known equation which describes the impact interaction of colliding elastic bodies according to the Hertz theory [33].

If $e \neq 0$, then since $\alpha$ is a small value, (108) could be rewritten in the form

$$
\ddot{\alpha}+a_{1} \dot{\alpha}=\frac{k_{\infty} e}{g^{2}}\left(\alpha^{2}\right) \cdot-\frac{k_{\infty}}{m} \alpha^{3 / 2} .
$$

Let us put $\alpha=V_{0} t$ in the right-hand side of (112) and integrate it. As a result we obtain

$$
\begin{aligned}
& \dot{\alpha}=V_{0}-a_{1} V_{0} t+k_{\infty} e g^{-2} V_{0}^{2} t^{2}, \\
& \alpha=V_{0} t-\frac{1}{2} a_{1} V_{0} t^{2}+\frac{1}{3} k_{\infty} e g^{-2} V_{0}^{2} t^{3} .
\end{aligned}
$$

From (113) we determine the refined duration of contact

$$
t_{\mathrm{reb}}^{0 e x}=t_{\mathrm{reb}}^{0}+\frac{2}{3} V_{0} \frac{e}{g}\left(t_{\mathrm{reb}}^{0}\right)^{2}
$$


where $t_{\text {reb }}^{0}=2 g k_{\infty}^{-1}$, as well as the refined time at which $\alpha$ attains its maximal value

$$
t_{\max }^{0 e x}=t_{\max }^{0}+\frac{e}{g} V_{0}\left(t_{\max }^{0}\right)^{2}
$$

where $t_{\max }^{0}=g k_{\infty}^{-1}$, and the maximal indentation

$$
\alpha_{\max }^{0 e x}=\alpha_{\max }^{0}+\frac{1}{3} V_{0}^{2} \frac{k_{\mathrm{\infty}} e}{g^{2}}\left(t_{\max }^{0}\right)^{3},
$$

where $\alpha_{\max }^{0}=(1 / 2) V_{0} g k_{\infty}^{-1}$.

5.2. The Case $\gamma=1$. At $\gamma=1,(101)$ and (102) after eliminating the inertia of the contact domain due to its smallness and considering that $\alpha=V_{0} t$ take the form

$$
\begin{aligned}
& \alpha+w \\
& \quad=V_{0} t-\frac{k_{\infty}}{m} V_{0}^{3 / 2} \int_{0}^{t}\left(t^{\prime 3 / 2}-\frac{2}{5} \Delta t^{\prime 5 / 2}\right)\left(t-t^{\prime}\right) d t^{\prime}, \\
& (e \alpha+g) \alpha^{1 / 2} W=k_{\infty} V_{0}^{3 / 2}\left(t^{3 / 2}-\frac{2}{5} \Delta t^{5 / 2}\right),
\end{aligned}
$$

where $\Delta=\sum_{j=1}^{4} M_{j} T_{j}^{-1}$, or after eliminating the value $W$ from (117)

$$
\begin{aligned}
& \dot{\alpha}=V_{0}-\frac{k_{\mathrm{\infty}}}{g} V_{0} t+\frac{k_{\mathrm{\infty}}}{g} V_{0}\left(\frac{2}{5} \Delta+\frac{e}{g} V_{0}\right) t^{2}, \\
& \alpha=V_{0} t-\frac{k_{\infty}}{2 g} V_{0} t^{2}+\frac{k_{\infty}}{3 g} V_{0}\left(\frac{2}{5} \Delta+\frac{e}{g} V_{0}\right) t^{3} .
\end{aligned}
$$

From (118) we find the refined contact duration

$$
t_{\mathrm{reb}}^{1 e x}=t_{\mathrm{reb}}^{0}+\frac{2}{3}\left(\frac{2}{5} \Delta+V_{0} \frac{e}{g}\right)\left(t_{\mathrm{reb}}^{0}\right)^{2}
$$

as well as the refined time at which $\alpha$ attains its maximal value

$$
t_{\max }^{1 e x}=t_{\max }^{0}+\left(\frac{2}{5} \Delta+V_{0} \frac{e}{g}\right)\left(t_{\max }^{0}\right)^{3}
$$

and the maximal indentation

$$
\alpha_{\max }^{1 e x}=\alpha_{\max }^{0}+\frac{1}{3} V_{0} \frac{k_{\infty}}{g}\left(\frac{2}{5} \Delta+V_{0} \frac{e}{g}\right)\left(t_{\max }^{0}\right)^{3} .
$$

Comparing formulas (114) and (119), (115) and (120), as well as (116) and (121), the following conclusion could be made: all characteristic values calculated at $\gamma=1$ are larger than those calculated at $\gamma=0$, and all characteristic values calculated at other magnitudes of $\gamma(0<\gamma<1)$ locate between these two corresponding limiting values in order of increasing magnitudes of the value $\gamma$.

\section{Appendix}

Let us consider for simplicity the dynamic behaviour of a viscoelastic rod which is described by a set of equations

$$
\begin{aligned}
& \frac{\partial \sigma}{\partial x}=\rho \frac{\partial^{2} u}{\partial t^{2}}, \\
& \frac{\partial u}{\partial x}=J_{\infty} \sigma+\int_{0}^{t} J^{(1)}\left(t-t^{\prime}\right) \sigma\left(x, t^{\prime}\right) d t^{\prime},
\end{aligned}
$$

where $\sigma$ is the stress, $u$ is the displacement, $\rho$ is the density, $t$ is the time, $J_{\infty}$ is the nonrelaxed magnitude of compliance, $J^{n}(t)=d^{n} J / d t^{n}, J(t)$ is the creep function, and $J^{(1)}=d J / d t$ is the creep kernel.

If we apply the condition of compatibility (11) to (A.1) and (A.2) and utilize to (A.2) the procedure adopted in the present paper for the deduction of relationships (65) and (66), then as a result we obtain from (A.1) the Hadamard dynamic condition of compatibility

$$
[\sigma]=-\rho G[v]
$$

and from the rheological equation (A.2) the relationship

$$
G[\sigma]=-E_{\infty}[v],
$$

where $E_{\infty}=J_{\infty}^{-1}$ is the nonrelaxed elastic modulus, and $v=$ $\partial u / \partial t$

Eliminating the value $[\sigma]$ from (A.3) and (A.4), we have

$$
G=\sqrt{\frac{E_{\infty}}{\rho}} .
$$

If one considers a pure elastic rod, then the same formulas (A.3)-(A.5) could be obtained, with the only difference that, for the elastic rod, as distinct from a viscoelastic one, there exists only one elastic modulus.

From the physical point of view, such a coincidence could be explained by the fact that discontinuities of the desired values on the front of the shock wave proceed very quickly; therefore on the wave front a viscoelastic material behaves itself as an elastic with the nonrelaxed modulus of elasticity.

Another matter if besides the interconnection between the discontinuities in stress and velocity it is necessary to find the time-dependence of the discontinuities $\left[\partial^{n} \sigma / \partial t^{n}\right]$ and $\left[\partial^{n} v / \partial t^{n}\right]$. In this case it is a need to differentiate (A.1) and (A.2) $n$ times and $n+1$ with respect to time $t$, respectively, and utilize the compatibility condition (11) wherein $Z$ is substituted by $\partial^{n-1} Z / \partial t^{n-1}$, resulting in the following condition of compatibility:

$$
G\left[\frac{\partial^{n} Z}{\partial x \partial t^{n-1}}\right]=-\left[\frac{\partial^{n} Z}{\partial t^{n}}\right]+\frac{\delta}{\delta t}\left[\frac{\partial^{n-1} Z}{\partial t^{n-1}}\right],
$$

$$
(n \geq 1) \text {. }
$$

Eliminating then the values $\left[\partial^{n} v / \partial t^{n}\right](n \geq 1)$ from the equations obtained after such a procedure, we are led to the recurrent equations $[34,35]$ 


$$
\begin{gathered}
\frac{\delta}{\delta t}\left[\frac{\partial^{n} \sigma}{\partial t^{n}}\right]+\alpha_{1}\left[\frac{\partial^{n} \sigma}{\partial t^{n}}\right]=F_{n-1}(t), \\
F_{n-1}(t)=\frac{1}{2} \frac{\delta^{2}}{\delta t^{2}}\left[\frac{\partial^{n-1} \sigma}{\partial t^{n-1}}\right]-\sum_{i=1}^{n} \alpha_{i+1}\left[\frac{\partial^{n-i} \sigma}{\partial t^{n-i}}\right], \alpha_{i}=\frac{J_{0}^{(i)}}{2 J_{0}}, J_{0}^{(i)}=J^{(i)}(0), J_{0}=J(0), \\
{\left[\frac{\partial^{n} v}{\partial t^{n}}\right]=-\frac{1}{\rho G}\left\{\left[\frac{\partial^{n} \sigma}{\partial t^{n}}\right]-\frac{\delta}{\delta t}\left[\frac{\partial^{n-1} \sigma}{\partial t^{n-1}}\right]\right\}, \quad(n \geq 0) .}
\end{gathered}
$$

Note that the discontinuity in the displacement $u$ is equal to zero, that is, $[u]=0$, since the medium is considered to be continuous without any cracks or damage. In other words, the function $u(t)$ could not be connected with the Heaviside function $H(t)$, as the Heaviside function assumes discontinuities, while the function $u(t)$ is continuous and does not experience any discontinuities with time.

Integrating (A.7) yields

$$
\begin{aligned}
{\left[\frac{\partial^{n} \sigma}{\partial t^{n}}\right]=} & \exp \left(-\alpha_{1} t\right) \int_{0}^{t} F_{n-1}\left(t^{\prime}\right) \exp \left(\alpha_{1} t^{\prime}\right) d t^{\prime} \\
& +A_{n} \exp \left(-\alpha_{1} t\right),
\end{aligned}
$$

where

$$
A_{n}=\left.\frac{\delta}{\delta t}\left[\frac{\partial^{n-1} \sigma}{\partial t^{n-1}}\right]\right|_{t=0}-\rho G v_{n}
$$

Constants $v_{n}$ are assigned on the rod's boundary $x=0$ due to the condition

$$
v(0, t)=\sum_{n=0}^{\infty} \frac{1}{n !} v_{n} t^{n}
$$

From (A.9) at $n=0$ we find the zero-order term of the ray series for the viscoelastic rod

$$
[\sigma]=A_{0} \exp \left(-\alpha_{1} t\right) H\left(t-x G^{-1}\right),
$$

while for the elastic rod we have

$$
[\sigma]=A_{0} H\left(t-x G^{-1}\right) .
$$

Equation (A.8) at $n=0$ is reduced to the dynamic condition of compatibility for the rod in the form of relationship (A.3).

In more intricate cases such as propagation of cylindrical wave-strips in viscoelastic plates, the discontinuities in desired values are proportional to $r^{-1 / 2} \exp (-\alpha t) H\left(t-r G^{-1}\right)$, where $r=r_{0}+G t$, and $r_{0}$ is the initial radius of the nonstationary cylindrical wave-strip (see, e.g., [36]).

Since in the present paper there is no need to find the time-dependence of the zeroth-order discontinuities, then we have restricted ourselves by the deduction of the dynamic conditions of compatibility (15) and (16) connecting the discontinuities in the generalized forces with the discontinuities in the generalized velocities, and these formulae differ in nothing in regard to the form from those for an elastic plate.

\section{Disclosure}

Phan Thanh Trung is on leave from HCMC University of Technology and Education, Vietnam.

\section{Competing Interests}

The authors declare that there is no conflict of interests regarding the publication of this paper.

\section{Acknowledgments}

This research was supported by the Ministry Education and Science of the Russian Federation.

\section{References}

[1] K. Diethelm, The Analysis of Fractional Differential Equations, Springer, Berlin, Germany, 2010.

[2] F. Mainardi, Fractional Calculus and Waves in Linear Viscoelasticity, Imperial College Press, London, UK, 2010.

[3] V. E. Tarasov, Fractional Dynamics: Applications of Fractional Calculus to Dynamics of Particles, Fields and Media, Higher Education Press, Beijing, China, 2010.

[4] J. A. Tenreiro Machado, M. F. Silva, R. S. Barbosa et al., "Some applications of fractional calculus in engineering," Mathematical Problems in Engineering, vol. 2010, Article ID 639801, 34 pages, 2010.

[5] R. Herrmann, Fractional Calculus: An Introduction for Physicists, World Scientific, New Jersey, NJ, USA, 2011.

[6] V. V. Uchaikin, Fractional Derivatives for Physicists and Engineers, vol. 2 of Vol.1. Background and Theory. Vol. 2. Applications, Springer, Berlin, Germany, Higher Education Press, Beijing, China, 2013.

[7] Y. Zhou, Basic Theory of Fractional Differential Equations, World Scientific, Singapore, 2014.

[8] J. A. Tenreiro Machado, A. M. S. F. Galhano, and J. J. Trujillo, "On development of fractional calculus during the last fifty years," Scientometrics, vol. 98, no. 1, pp. 577-582, 2014.

[9] C. Cattani, H. M. Srivastava, and X.-J. Yang, Fractional Dynamics, De Gruyter Open, 2016.

[10] F. Zeng and C. Li, Numerical Methods for Fractional Calculus, CRC Press, Boca Raton, Fla, USA, 2015.

[11] R. Daou and M. Xavier, Eds., Fractional Calculus: History, Theory and Applications, Nova Science Publishers, New York, NY, USA, 2015. 
[12] Y. A. Rossikhin and M. V. Shitikova, "Application of fractional calculus for dynamic problems of solid mechanics: novel trends and recent results," Applied Mechanics Reviews, vol. 63, no. 1, Article ID 010801, 52 pages, 2010.

[13] Y. A. Rossikhin and M. V. Shitikova, "Two approaches for studying the impact response of viscoelastic engineering systems: an overview," Computers \& Mathematics with Applications, vol. 66, no. 5, pp. 755-773, 2013.

[14] D. Ingman and J. Suzdalnitsky, "Response of viscoelastic plate to impact," Journal of Vibration and Acoustics, vol. 130, no. 1, Article ID 011010, 8 pages, 2008.

[15] Y. A. Rossikhin and M. V. Shitikova, "Dynamic response of a viscoelastic plate impacted by an elastic rod," Journal of Vibration and Control, vol. 22, no. 8, pp. 2019-2031, 2016.

[16] Y. A. Rossikhin and M. V. Shitikova, "The analysis of the impact response of a thin plate via fractional derivative standard linear solid model," Journal of Sound and Vibration, vol. 330, no. 9, pp. 1985-2003, 2011.

[17] Y. A. Rossikhin and M. V. Shitikova, "Fractional-derivative viscoelastic model of the shock interaction of a rigid body with a plate," Journal of Engineering Mathematics, vol. 60, no. 1, pp. 101-113, 2008.

[18] J. A. Zukas, T. Nicholas, H. F. Swift, L. B. Greszchuk, and D. R. Curran, Impact Dynamics, John Wiley \& Sons, New York, NY, USA, 1982.

[19] Y. A. Rossikhin and M. V. Shitikova, "The impact of elastic bodies upon beams and plates with consideration for the transverse deformations and extension of a middle surface," ZAMM Zeitschrift fur Angewandte Mathematik und Mechanik, vol. 76, supplement 5, pp. 433-434, 1996.

[20] Yu. A. Rossikhin, M. V. Shitikova, and M. G. Estrada Meza, "Impact response of a Timoshenko-type viscoelastic beam considering the extension of its middle surface," SpringerPlus, vol. 5, article 206, 2016.

[21] Y. A. Rossikhin, M. V. Shitikova, and I. I. Popov, "Dynamic response of a viscoelastic beam impacted by a viscoelastic sphere," Computers and Mathematics with Applications, 2017.

[22] I. I. Popov, T.-P. Chang, Y. A. Rossikhin, and M. V. Shitikova, "Experimental study of concrete aging effect on the contact force and contact time during the impact interaction of an elastic rod with a viscoelastic beam," Journal of Mechanics, 2017.

[23] L. Merrill, J. Sauer, and A. Woodward, "Dynamic mechanical studies of irradiated polyethylene," Polymer, vol. 1, pp. 351-364, 1960.

[24] I. I. Popov, Y. A. Rossikhin, M. V. Shitikova, and T.-P. Chang, "Impact response of a viscoelastic beam considering the changes of its microstructure in the contact domain," Mechanics of Time-Dependent Materials, vol. 19, no. 4, pp. 455-481, 2015.

[25] Y. A. Rossikhin and M. V. Shitikova, "A ray method of solving problems connected with a shock interaction," Acta Mechanica, vol. 102, no. 1-4, pp. 103-121, 1994.

[26] Y. A. Rossikhin and M. V. Shitikova, "The ray method for solving boundary problems of wave dynamics for bodies having curvilinear anisotropy," Acta Mechanica, vol. 109, no. 1-4, pp. 49-64, 1995.

[27] T. Y. Thomas, Plastic Flow and Fracture in Solids, Mathematics in Science and Engineering, Vol. 2, Academic Press, New York, NY, USA, 1961.

[28] Yu. A. Rossikhin and M. V. Shitikova, "Ray expansion theory," in Encyclopedia of Thermal Stresses, vol. 8, pp. 4108-4131, Springer, Berlin, Germany, 2014.
[29] Y. N. Rabotnov, Elements of Hereditary Solid Mechanics, Nauka, Moscow, Russia, 1977, English Translation by Mir Publishers, Moscow, Russia, 1980.

[30] Y. N. Rabotnov, "Equilibrium of an elastic medium with aftereffect," Fractional Calculus and Applied Analysis, vol. 17, no. 3, pp. 684-696, 2014.

[31] E. Kamke, Differentialgleichungen Lösungsmethoden und Lösungen, Akademische Verlagsgesellschaft, Leipzig, Germany, 1943.

[32] Y. A. Rossikhin and M. V. Shitikova, "Transient response of thin bodies subjected to impact: wave approach," The Shock and Vibration Digest, vol. 39, no. 4, pp. 273-309, 2007.

[33] A. E. H. Love, A Treatise on the Mathematical Theory of Elasticity, Cambridge University Press, Cambridge, UK, 1906.

[34] J. D. Achenbach and D. P. Reddy, "Note on wave propagation in linearly viscoelastic media," Zeitschrift für angewandte Mathematik und Physik, vol. 18, no. 1, pp. 141-144, 1967.

[35] Yu. A. Rossikhin and M. V. Shitikova, "To the construction of uniformly valid forward-area asymptotics in terms of the ray method in dynamic problems in linear viscoelasticity," ASME Journal of Applied Mechanics, vol. 61, no. 3, pp. 744-746, 1994.

[36] Y. A. Rossikhin, M. V. Shitikova, and A. A. Loktev, "Viscoelastic transverse impact of a sphere on an Uflyand-Mindlin plate," in Proceedings of the 8th International Conference on Computational Plasticity: Fundamentals and Applications (COMPLAS '05), E. Onate and D. R. J. Owen, Eds., pp. 372-375, Barcelona, Spain, September 2005. 


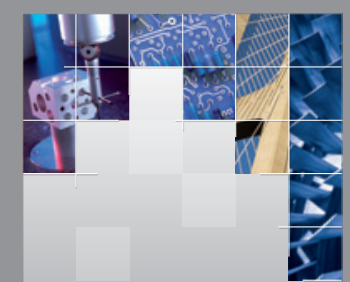

\section{Enfincering}
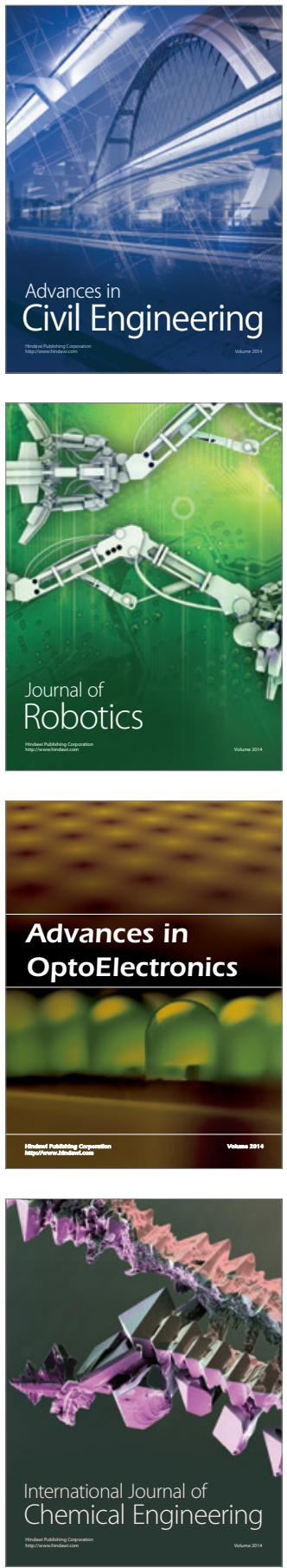

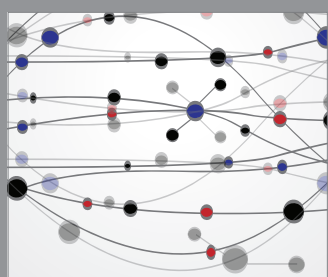

The Scientific World Journal

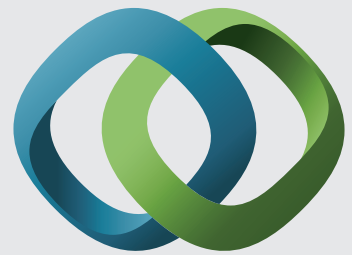

\section{Hindawi}

Submit your manuscripts at

https://www.hindawi.com
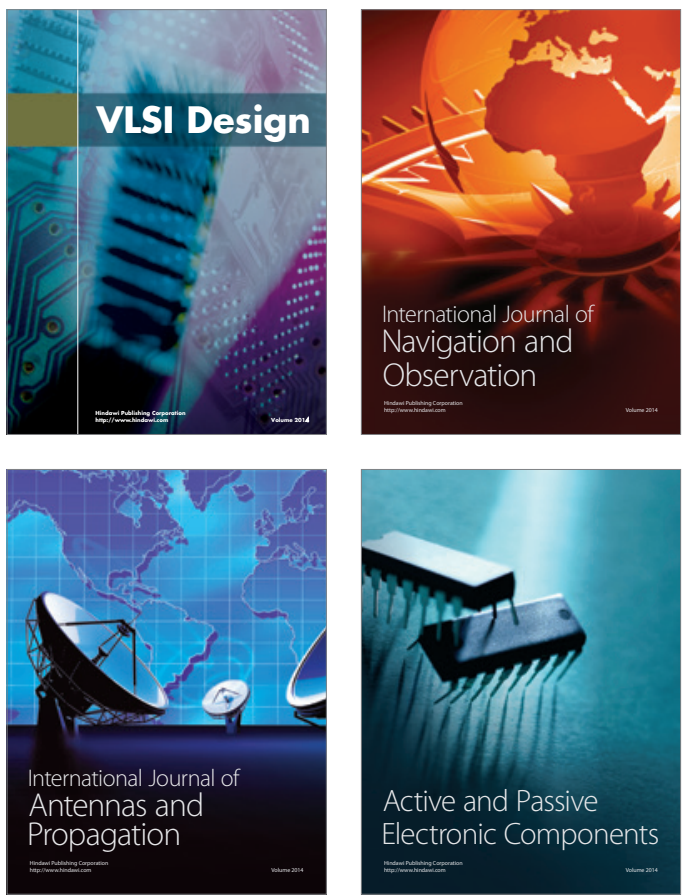
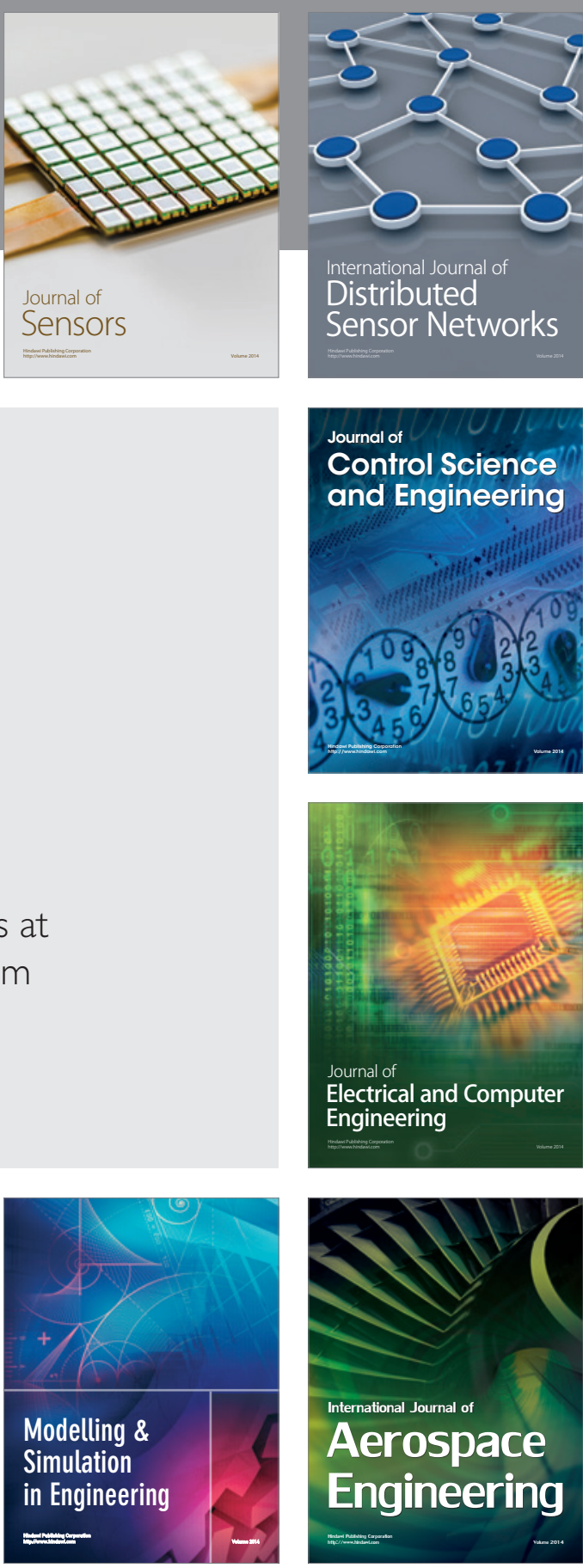

International Journal of

Distributed

Sensor Networks

$-$

Joumal of

Control Science

and Engineering
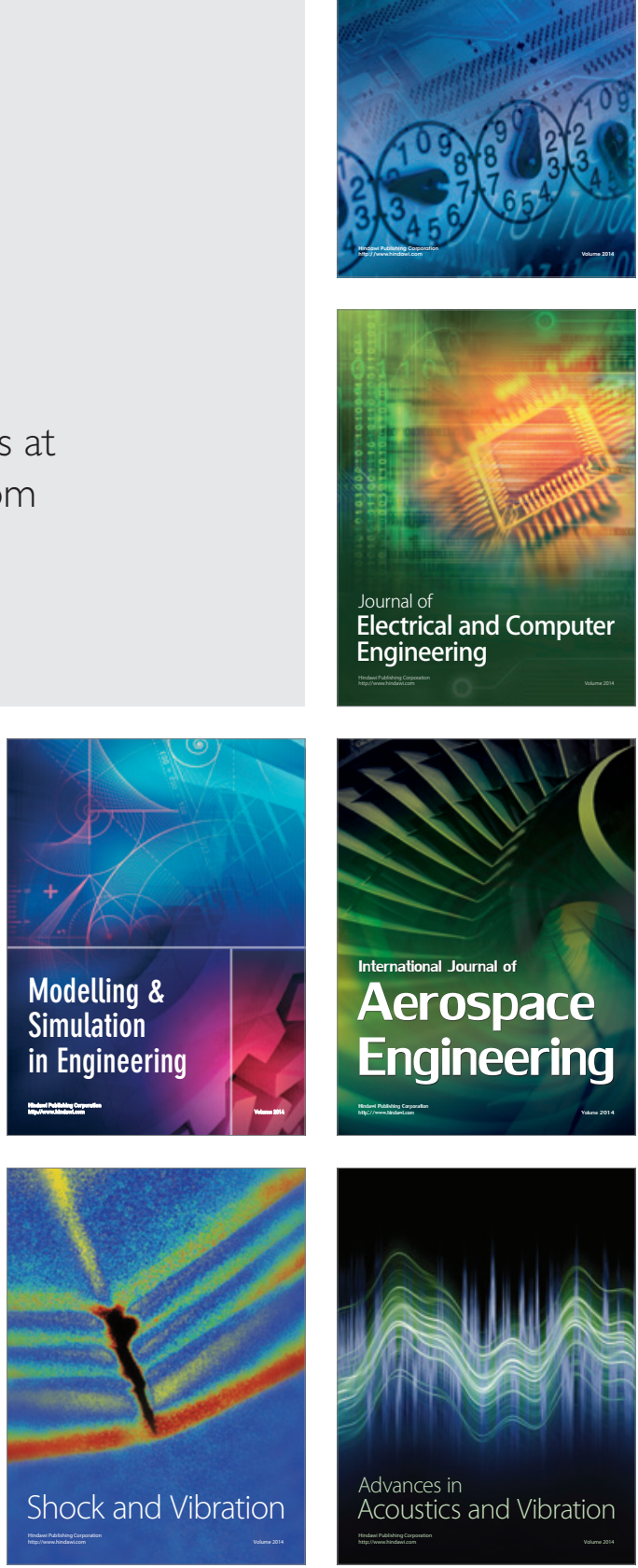